\title{
Acoustical Semi-blind Deconvolution for Bearing Defect Detection
}

\author{
Yu Wang ${ }^{1, a}$, Wen Zhong ${ }^{1}$, Yang Yin ${ }^{1}$, Xiaoyin Liu ${ }^{1}$ and Chunhua Song ${ }^{1}$ \\ ${ }^{1}$ Xihua University, School of Mechanical Engineering, Chengdu, China
}

\begin{abstract}
Acoustical machine monitoring is frequently complicated by noisy environments at a production site. This paper presents a semi-blind deconvolution algorithm to extract only one desired acoustic source signal from different sources which are convoluted and mixed by mechanical systems before being measured. The method is based on blind model transformation, robust independent component analysis, reference signal and spectral distance. The new algorithm is tested on simulation and experimental cases. Results demonstrate that blind separation of acoustic signals is possible even when measurements are distanced from vibration exciting sources of faulty bearings. Furthermore, the method can eliminate the effect of structural resonances and large reverberation time of mixtures, which often causes severe problems in classical acoustical diagnostic methods of rolling element bearings.
\end{abstract}

\section{Introduction}

Machine faults modify machine sounds characteristically, therefore observing machine sounds can be a useful mean for condition monitoring and fault diagnosis [1]. Acoustical diagnosis utilizes acoustic signals which are detected by microphones, so non-contact detection and easy handling are excellent features of this diagnosis [2]. However, it is hard to apply the acoustic diagnosis to the precise diagnosis, because the machine sound from the diagnosed part is usually corrupted by other interfering sources and non-directive noise, which is often much louder. Therefore, signal processing techniques such a blind source separation (BSS) should be employed as a preprocessor to increase the portion of relevant information in the measured signal [3]. Due to timedelayed superposition of the sources and reflections from the walls, acoustical mixtures have to be modeled with convolutive mixtures [4]. Existing BSS methods are not well geared to handle such situations, because the modifying paths of vibrations (dynamics of the system) and propagation paths of sound (in air) are neither controllable nor known $[5,6]$. We show a modified blind deconvolution method, enabling to tackle problems of both multiple convoluted sources and monitoring measurements, as it uses an analytically derived spectral distance in the optimization process of the cost function used. As a consequence, this method possesses a good behaviour when the reverberation time of mixtures is sufficiently large and the machine is operating in a noisy environment.

The paper is organized as following: The basic problem is described in Section 2. Section 3 presents the proposed method. Section 4 and Section 5 show the numerical simulation, experimental setup and results of the separation procedure. Conclusions are presented in Section 6.

\section{Problem formulation}

In the following, all signals are assumed of stochastic, zero-mean and finite-power. At a discrete time index $t$, a convolutive mixing process is usually described as a linear Multiple-Input Multiple-Output (MIMO) system:

$$
x_{i}(t)=\sum_{j=1}^{n} \sum_{\tau=0}^{K_{i j}} h_{i j}(\tau) s_{j}(t-\tau), i=1, \cdots, m(m \geq n)
$$

where $\mathbf{x}(t)=\left[x_{1}(t), \cdots, x_{m}(t)\right]^{\mathrm{T}}$ denotes observed signals from an array of $m$ microphones, $\mathbf{s}(t)=\left[s_{1}(t), \cdots, s_{n}(t)\right]^{\mathrm{T}}$ denotes $n$ unknown original source signals, $[\cdot]^{\mathrm{T}}$ denotes a transposition of a vector or a matrix, $h_{i j}$ is impulse responses of a system (mixing filters), and $K_{i j}$ denotes the corresponding length of $h_{i j}$ which may change in time.

For brevity, the convolutive mixing model Eq.(1) can be written in a following matrix form:

$$
\mathbf{x}(t)=\sum_{\tau=0}^{K} \mathbf{H}_{\tau} \mathbf{s}(t-\tau)
$$

where $\mathbf{H}_{\tau}$ is an $m \times n$ mixing matrix which contains the $\tau$-th filter coefficients.

Assuming that all source signals arrive at sensors or microphones at the same time without being filtered, the convolutive mixture model Eq.(2) simplifies to a instantaneous mixture model:

$$
\mathbf{x}(t)=\mathbf{H s}(t)
$$

\footnotetext{
$\overline{{ }^{\mathrm{a}} \text { Corresponding author: redtu160@ } 163 . c o m}$
} 
where $\mathbf{H}$ is an $m \times n$ matrix containing mixing coefficients.

The goal of the classical BSS is to find an $m \times n$ unmixing matrix $\mathbf{W}$ to recover all sources:

$$
\mathbf{y}(t)=\mathbf{W x}(t)=\mathbf{W H s}(t)=\mathbf{P D s}(t)
$$

where $\mathbf{P} \in \mathbf{R}^{m \times m}$ is a permutation matrix, and $\mathbf{D} \in \mathbf{R}^{m \times m}$ is a diagonal scaling matrix.

In contrast to most classical BSS algorithms, the constrained independent component analysis (cICA) algorithm does not need recover all sources. Furthermore, its goal is to recover only one source. For extracting one independent source signal, a reliable and flexible contrast function $J(y)$ of the cICA is defined by:

$$
\begin{gathered}
\max J(y) \approx \rho[\mathrm{E}\{G(y)\}-\mathrm{E}\{G(v)\}]^{2} \\
\text { s.t. } g(\mathbf{w})=\varepsilon(y, r)-\xi \leq 0, \quad h(\mathbf{w})=\mathrm{E}\left\{y^{2}\right\}-1=0
\end{gathered}
$$

where $\rho$ is a positive constant, $v$ is a Gaussian variable having zero mean and unity variance, $G(\bullet)$ is a nonquadratic function. The equality constraint $h(\mathbf{w})$ ensures that the contrast function $J(y)$ and the weight vector $\mathbf{w}$ are bounded. $\varepsilon(y, r)$ is the closeness measure between the extracted signal $y$ and the reference signal $r, \xi$ is a threshold, and $\mathrm{E}\{\boldsymbol{\bullet}\}$ denotes a sample mean operator. From the contrast function Eq.(5) and Eq.(6), $\mathrm{Lu}$ and Rajapakse derived the cICA algorithm $[7,8]$ :

$$
\begin{gathered}
\mathbf{w}_{k+1}=\mathbf{w}_{k}-\eta \mathbf{R}_{\mathbf{x x}}^{-1} L_{\mathbf{w}_{k}}^{\prime} / \delta\left(\mathbf{w}_{k}\right) \\
L_{\mathbf{w}_{k}}^{\prime}=\bar{\rho} \mathrm{E}\left\{\mathbf{x} G_{y}^{\prime}(y)\right\}-0.5 \mu \mathrm{E}\left\{\mathbf{x} g_{y}^{\prime}\left(\mathbf{w}_{k}\right)\right\}-\lambda \mathrm{E}\{\mathbf{x} y\} \\
\delta\left(\mathbf{w}_{k}\right)=\bar{\rho} \mathrm{E}\left\{\mathbf{x} G_{y^{2}}^{\prime \prime}(y)\right\}-0.5 \mu \mathrm{E}\left\{g_{y^{2}}^{\prime \prime}\left(\mathbf{w}_{k}\right)\right\}-\lambda
\end{gathered}
$$

where $k$ denotes the iteration index, $\eta$ is the learning rate, $\mathbf{R}_{\mathbf{x x}}$ is the covariance matrix of the observed signals $\mathbf{x}, \bar{\rho}=\rho \cdot \operatorname{sign}(E\{G(y)\}-E\{G(v)\}), G_{y}^{\prime}(y)$ and $G_{y^{2}}^{\prime \prime}(y)$ are the first and the second derivatives of $G(y)$ with respect to $y$, and $g_{y}^{\prime}\left(\mathbf{w}_{k}\right)$ and $g_{y^{2}}^{\prime \prime}\left(\mathbf{w}_{k}\right)$ are the first and the second derivatives of $g\left(\mathbf{w}_{k}\right)$ with respect to $y$. The optimum multipliers $\mu$ and $\lambda$ are found by iteratively updating them based on a gradient-ascent method: $\mathbf{w}_{0} r(t) \mathbf{w}_{k+1}=\mathbf{w}_{k+1}^{+} /\left\|\mathbf{w}_{k+1}^{+}\right\|$

$$
\begin{array}{r}
\mu_{k+1}=\max \left\{0, \mu_{k}+\gamma g\left(\mathbf{w}_{k}\right)\right\} \\
\lambda_{k+1}=\lambda_{k}+\gamma h\left(\mathbf{w}_{k}\right)
\end{array}
$$

\section{Method development}

Unfortunately, there are two important issues that should be and solved when the cICA algorithm comes to mixed acoustic signals in a shop floor. One issue is that the cICA based on instantaneous mixture can not be applied to convolutive mixtures of sources, and another one is the design of the reference signal.

Hence, instead of modeling individual samples at a discrete time index $t, N$ successive samples of observation column vector are stacked to construct a new block. The equations for such a block can be written as follows:

$$
\begin{aligned}
& x(t)=h(0) s(t)+\cdots+h(K) s(t-K), \\
& x(t-1)=h(0) s(t-1)+\cdots+h(K) s(t-K-1), \cdots, \\
& x(t-N)=h(0) s(t-N)+\cdots+h(K) s(t-K-N)
\end{aligned}
$$

The $m$-dimensional observation sequence can be written as an $m N$-dimensional column vector $\widehat{\mathbf{x}}(t)$ in Eq.(13), and the $n$-dimensional source sequence can be written as an $n(N+K)$-dimensional vector in Eq.(14).

$$
\begin{aligned}
& \widehat{\mathbf{x}}(t)=\left[x^{\mathrm{T}}(t), x^{\mathrm{T}}(t-1), \cdots, x^{\mathrm{T}}(t-N+1)\right]^{\mathrm{T}} \\
& \widehat{\mathbf{s}}(t)=\left[s^{\mathrm{T}}(t), s^{\mathrm{T}}(t-1), \cdots, s^{\mathrm{T}}(t-N-K+1)\right]^{\mathrm{T}}
\end{aligned}
$$

where $x^{\mathrm{T}}(t)=\left[x_{1}(t), \cdots, x_{m}(t)\right]^{\mathrm{T}}, s^{\mathrm{T}}(t)=\left[s_{1}(t), \cdots, s_{n}(t)\right]^{\mathrm{T}}$.

Then, the convolutive mixing model Eq.(1) and Eq.(3) can be reformulated as [9]:

$$
\widehat{\mathbf{x}}(t)=\hat{\mathbf{H}} \widehat{\mathbf{s}}(t)
$$

where $\hat{\mathbf{H}}$ is a block-Toeplitz matrix and has dimensions $m N \times n(N+K)$ :

$$
\widehat{\mathbf{H}}=\left[\begin{array}{cccccccc}
h(0) & h(1) & \cdots & h(K) & 0 & \cdots & \cdots & 0 \\
0 & h(0) & h(1) & \cdots & h(K) & 0 & \cdots & 0 \\
& \vdots & & & & & & \vdots \\
0 & \cdots & \cdots & 0 & h(0) & h(1) & \cdots & h(K)
\end{array}\right]
$$

This equation is very interesting. It tells us Eq.(15) has the same structure as the instantaneous mixture model given in Eq.(3), and the task of blind convolutive model can be solved by the cICA algorithm based on instantaneous model.

However, the second issue arises accordingly, i.e. the design of the reference signal. James and Gibson pointed out that the shape, the phase and the occurrence time of the reference signal may influence the output result [10]. This cICA method requires that the desired source signal is strong enough to be observed in a sensor signal, which cannot be always satisfied. Consequently, in contrast to the conventional measure distance in the standard cICA algorithm, the closeness measure $\varepsilon(y, r)$ is newly defined as:

$$
\varepsilon(y, r)=\frac{1}{2 N} \sum_{f=0}^{N}\left[\frac{Y(f)}{R(f)}+\frac{R(f)}{Y(f)}\right]-1, \quad f=1,2, \cdots, N
$$

where $Y(f)$ and $R(f)$ are power spectra of $y(t)$ and $r(t)$ respectively.

Next, let us now show a valid approach to find the reference signal, whose phase and waveform do not have to be well matched to the desired source signal.

The simulation of the simplified signal model of rolling element bearing can be expressed as:

$$
s(t)=e^{-B(t-\Delta t)} \cos \left(2 \pi f_{n} t+\varphi\right)
$$

Here, the original phase $\varphi$ is assumed to be zero, and $B$ is an appropriate value. Assuming that there is a priori 
information about frequency available, a reference signal $r(t)$ can be easily obtained based on Eq.(18):

$$
r(t)=\operatorname{sign}\left(\sin \left(\pi f_{c} t\right)\right)
$$

where $\operatorname{sign}(\cdot)$ means sign operation, $f_{c}$ is a certain characteristic frequency.

Finally, the block diagram of the proposed algorithm is shown in Figure 1.

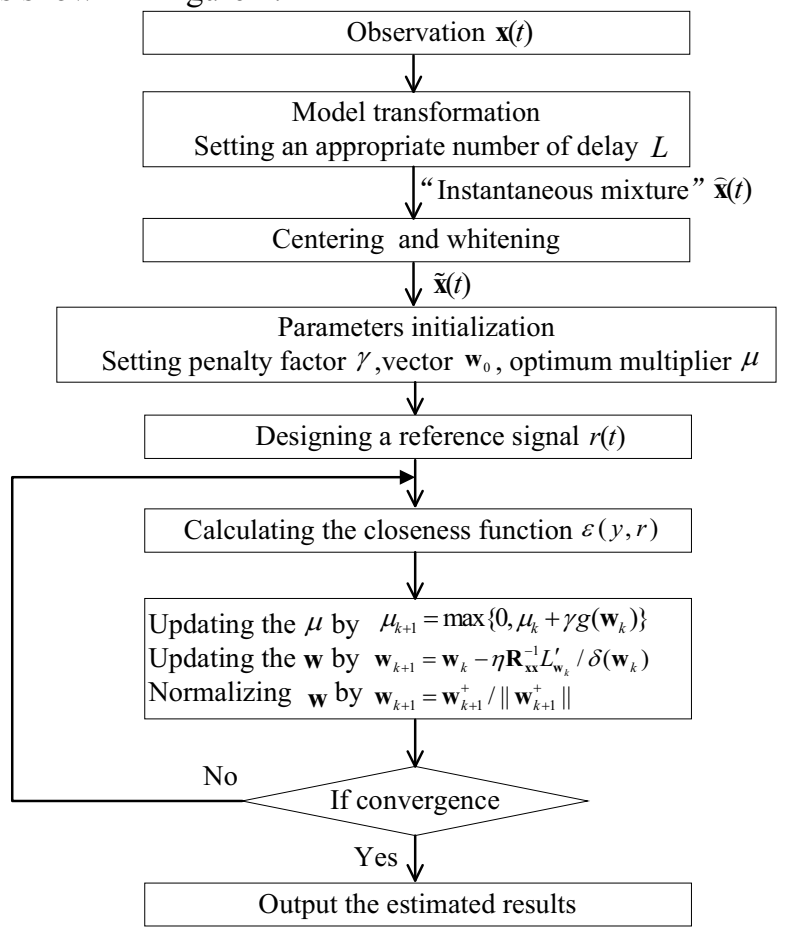

Figure 1. Algorithm flow chart for single source estimation

\section{Numerical simulation}

To verify the validity of the proposed algorithm, a computer simulation was carried out, in which two simulated signals of rolling bearings with outrace fault were created. First, three simulated source signals were generated as follows:

$s_{1}(t)=e^{-580(t-0.25)} \cos (2 \pi \cdot 2570 \bullet t)$

$s_{1}(t)=e^{-580(t-0.25)} \cos (2 \pi \cdot 2570 \bullet t)$

$s_{3}(t)=\sin (2 \pi \cdot 345 \bullet t)+\sin (2 \pi \cdot 2530 \bullet t)+\cos (2 \pi \cdot 2990 \bullet t)$

Here, the sample rate $f_{s}=8192 \mathrm{~Hz}$ and the sample points $n=8192$ (intervals between adjacent pulses: $s_{1}(t): 100$ sample points, $s_{2}(t): 176$ sample points). Second, each source signal was normalized to unit variance and had zero mean. Third, the convolutive mixing process is defined by Eq.(1) and the convolutive mixing matrix $h_{i j}(\tau)$ was generated randomly with length 128 in order to simulate the case where the timedelay is sufficiently large.

Figure 2. shows the source signals and their amplitude spectra. It can be seen that the first and second signals are typical of structural systems excited by impacts, i.e., periodic trains of decaying oscillations. These types of signals are often considered as models of faulty bearing vibrations. The third one is a periodic signal. Artificially, the mixtures were corrupted by additive white Gaussian noise, and signal to noise ratio (SNR) SNR1=7.5, SNR2=5.6 and SNR3=8.3 approximately. The waveforms and spectra of the mixed signals are shown in Figure 3. Intuitively, both the waveforms and the spectra of the mixed signals provide little information of the periodic impulses.
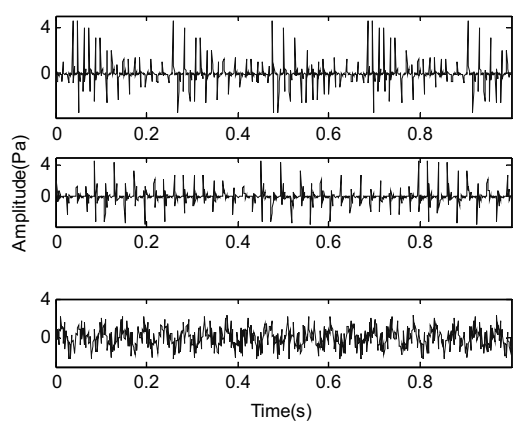

(a)
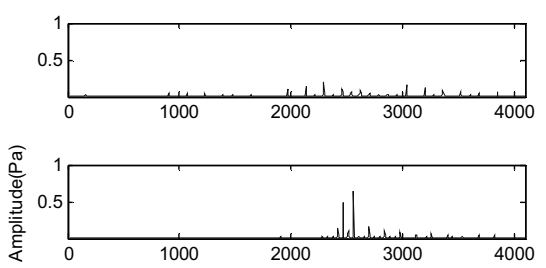

(b)

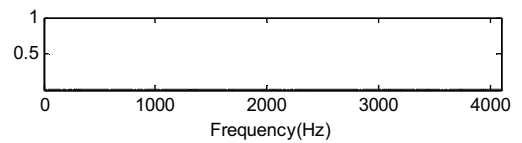

Figure 2. Simulated source signals (a) Time domain waveforms (b) Amplitude spectra

Next, the proposed algorithm was applied to recover the three source signals. Some information about the algorithm parameters is first given: $L=40$ and $G(u)=u^{2}$. The reference signal and estimated result are shown in Figure 4. and Figure 5. respectively. It can be seen that our method successfully separated the transient impulse signals and provides a very good result. This simulation demonstrates the effectiveness of the proposed algorithm. Nevertheless, we found that it could enhance the separation quality by increasing the filter length, but the computational complexity of the algorithm increases dramatically with the factor (without regard to sample points). It seems that further investigations are necessary concerning the effects of the parameter.
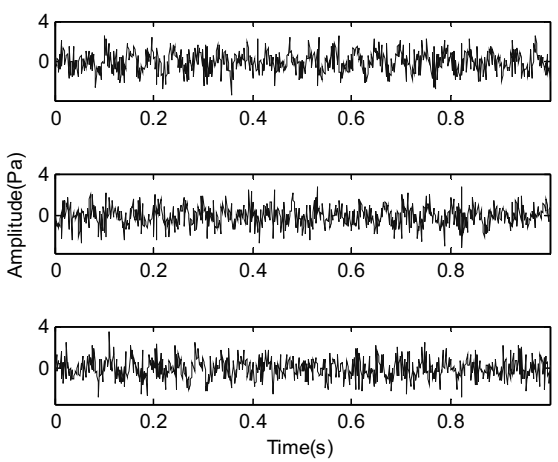

(a) 

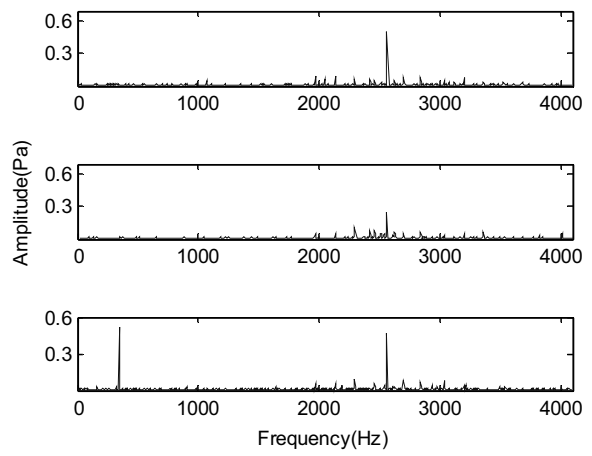

(b)

Figure 3. Simulated mixed signals (a) Time domain waveforms (b) Amplitude spectra

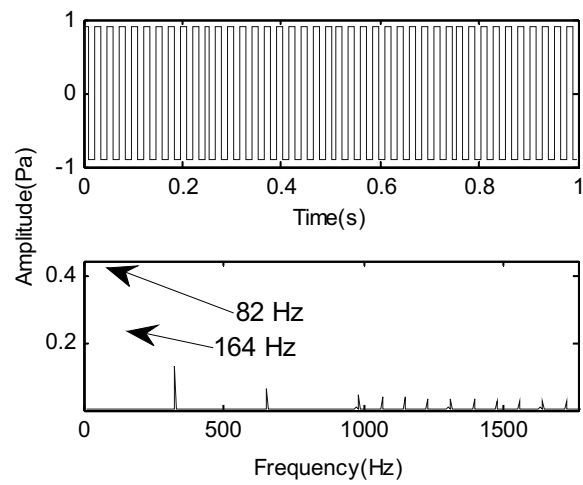

(a)

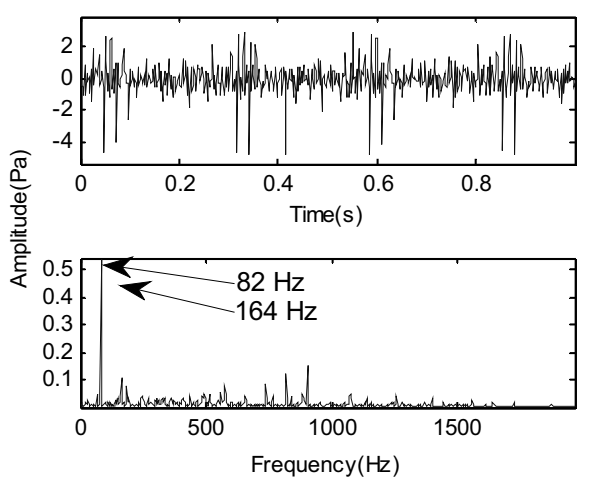

(b)

Figure 4. Time domain waveforms and envelope spectra (a) Simulated reference signal (b) Estimated signal (Characteristic frequency $82 \mathrm{~Hz}$ )

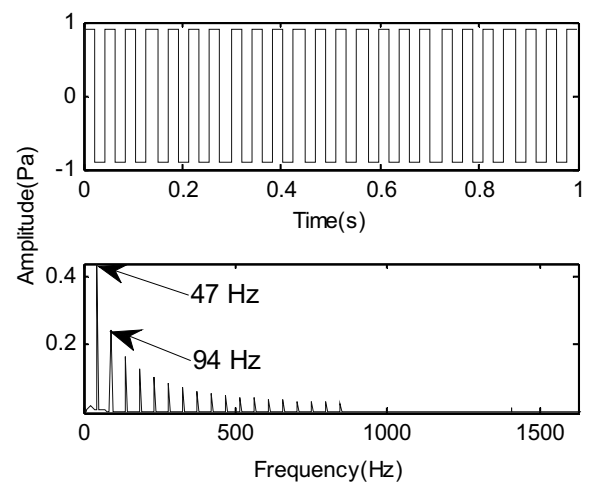

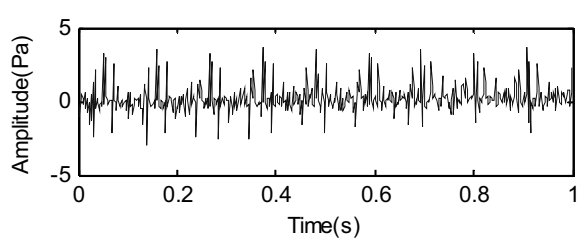

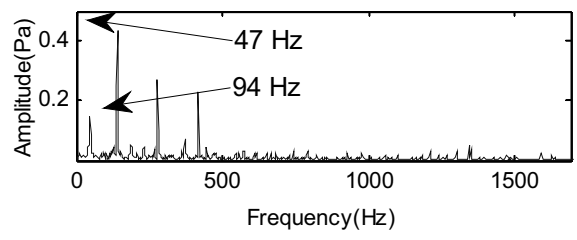

(b)

Figure 5. Time domain waveforms and envelope spectra (a) Simulated reference signal (b) Estimated signal (Characteristic frequency $47 \mathrm{~Hz}$ )

\section{Experimental setup and results}

To further investigate the effectiveness of the presented algorithm for the recovery of fault bearing acoustic signals, a test bed in our laboratory was built. The test bed was equipped with an electric motor with changeable rotation speed, which was connected with the main shaft of the system through a belt pulley. Two rolling bearings were used to mount the shaft. One rolling element bearing with an artificial defect in the outer race was tested. All acoustic signals were collected by two microphones in different positions, which are illustrated in Figure 6. Moreover, a small drilling machine was used to be an interference source and its sound is shown in Figure 7. The experiment was executed at $13.33 \mathrm{~Hz}$ rotation rate of shaft. The sound signals were captured at the sampling frequency of $8192 \mathrm{~Hz}$ and the sample points were 8192 .



Figure 6. Experimental setup



(a)

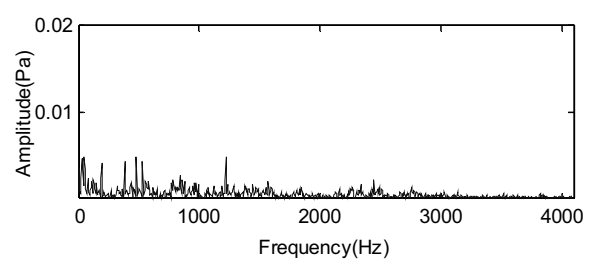

(b)

Figure 7. Acoustic signal of the drilling machine (a) Time domain waveform (b) Amplitude spectrum 
The signals in Figure 8. represent two raw sensor signals without any processing. It is seen that no impacts can be located. Fault related signatures are usually modulated by the signals at high resonance frequencies of the bearing and surrounding structures, thus a $1000 \mathrm{~Hz}$ cut-off frequency is selected in this work to eliminate the effects of low-frequency noisy components. In Figure 9., filtered signals using a high-pass filter (high pass frequency $f=1000 \mathrm{~Hz}$ ) are presented. Figure 10 . shows the envelope spectra of the filtered signals. The signature of the rolling element fault is not inspected through the filtered signals and their corresponding envelope spectra for and the actual outer race fault. Hence, the raw signals and filtered signals were heavily interfered by other undesired signals and can not to be utilized for bearing defect detection directly.

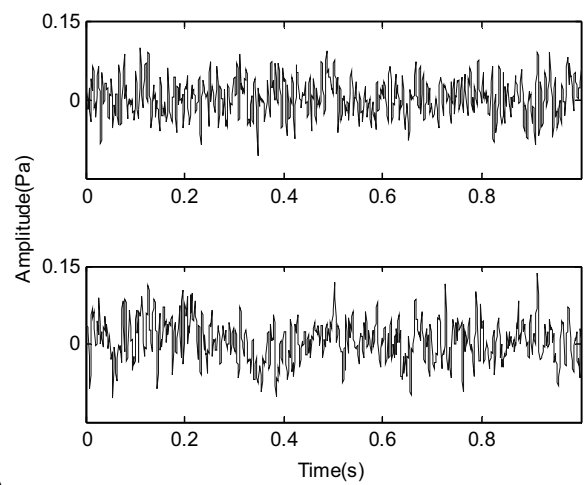

(a)

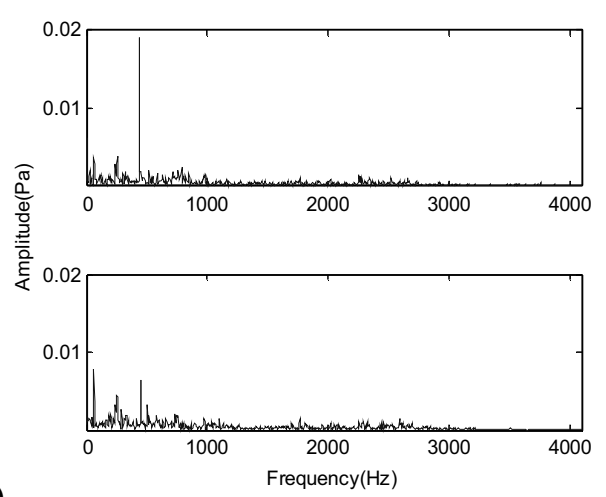

(b)

Figure 8. Sensor signals (a) Time domain waveforms (b) Amplitude spectra

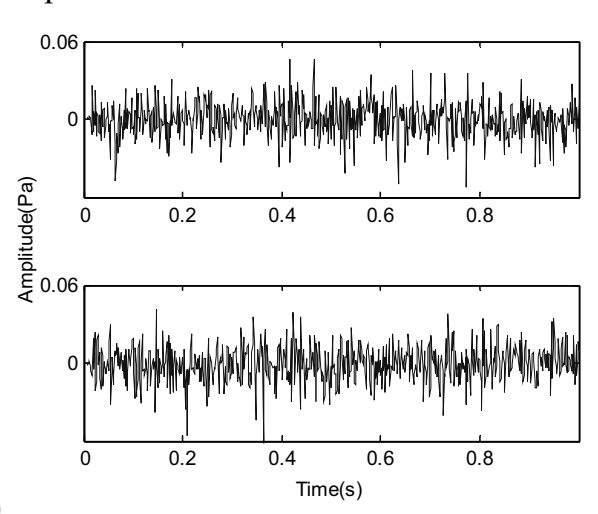

(a)

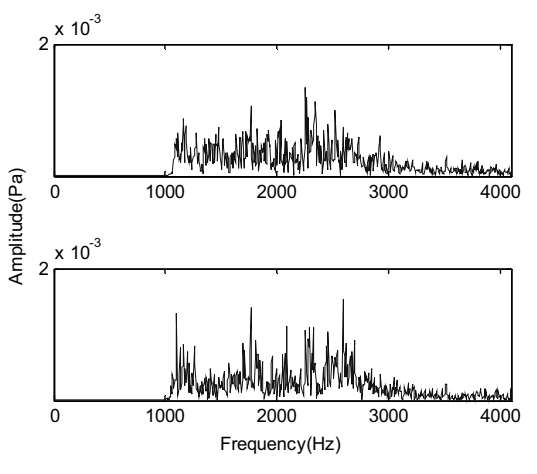

Figure 9. Filtered signals using a high-pass filter (cut-off frequency $f=1000 \mathrm{~Hz}$ ) (a) Time domain waveforms (b) Amplitude spectra

Then, the proposed algorithm was utilized herein to extract the source signal from sensor signals. The algorithm parameters are first given as follows: $L=30$ and $G(u)=u^{2}$. The estimated signal in time and frequency domains is presented in Figure 10. The kurtosis of the signal increased dramatically to 38.55 compared to -0.40 for the raw signal and 1.25 for the filtered signal. Intuitively, the recovered signal may come from the faulty bearing according to the periodic impulses. This idea will be confirmed based on the knowledge about the diagnosed bearing and its rotation speed (about $800 \mathrm{rpm}$ ). The parameters of the bearing are given as follows: Pitch diameter: $D=39 \mathrm{~mm}$, Ball diameter: $d=7.5 \mathrm{~mm}$, Number of rolling elements: $Z=12$, Contact angular: 0 .

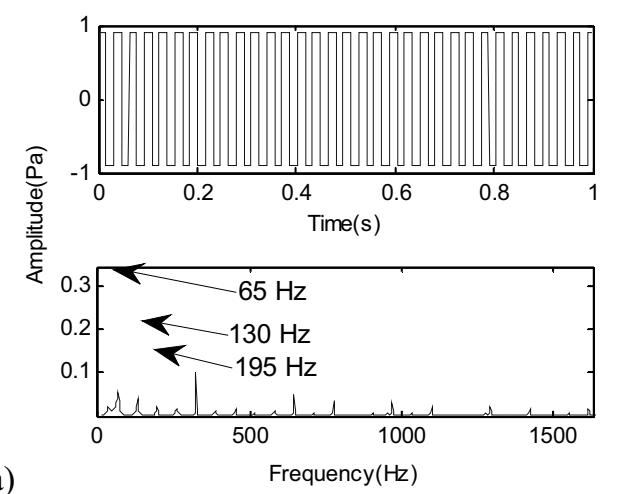

(a)

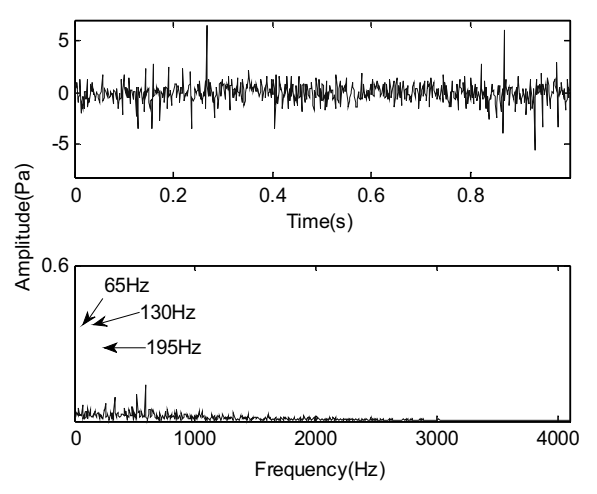

(b)

Figure 10. Time domain waveforms and envelope spectra (a) Reference signal (b) Estimated signal (Characteristic frequency $64.6 \mathrm{~Hz}$ ) 
It can be calculated that the characteristic frequency of defected outer race is $64.6 \mathrm{~Hz}$ theoretically. As can be viewed, the ball pass frequency of the outer race and its harmonics are all visible, and the analysis result $(65 \mathrm{~Hz})$ is very close to the calculated result $(64.6 \mathrm{~Hz})$. Hence, it is concluded that the impacts are confirmed to be generated by the defective bearing and the estimated signal in Figure 10. belongs to the defected bearing. The results of the experiment can be evaluated as satisfactory and demonstrate that the proposed algorithm can be implemented to extract acoustical impulse signals of faulty bearings.

It is also worth mentioning that the scaling ambiguity, permutation ambiguity, and delay ambiguity exist in the recovered results, but these ambiguities are not essential, since the most relevant information about the faults is contained in the waveforms or spectra of the source signals and not in their amplitudes, order or time-delays.

\section{Conclusions}

In this paper, we proposed a blind deconvolution method for detection the incipient fault of rolling bearings by acoustic analysis. The new approach has several advantages listed as below. First, the proposed algorithm can perform successfully in complicated acoustic situations with low computational cost even when the time-delay of mixtures is very large. Second, it also does not require the knowledge of the number of sources like in classical BSP (the number of sensors is greater than the number of the sources) and it works well in the presence of multiple additive noise. Experiments with simulated signals and real machine sound from a test bed and drill machine in a room are conducted to demonstrate the effectiveness of this algorithm. The results show that this algorithm can be efficiently applied to rolling element bearing fault detection in practice, and is very promising in acoustic-based machine diagnosis.

\section{Acknowledgements}

This work is supported by the National Natural Science Foundation of China (Approved Grant: 51305357) and Scientific Research Fund of Sichuan Provincial Education Department (Approved Grant: 13ZB0018). The authors are also grateful to the Open Research Fund of Key Laboratory of Manufacturing and Automation, Xihua University (SZJJ2012-024) for funding this discovery project.

\section{References}

1. M. Knaak, M. Kunter, D. Filberi, 14th International Conference on Digital Signal Processing, 1 (2002)

2. R. M. Vilela, J.C. MetrGlho, J.C. Cardoso, Proceedings of the 12th IEEE Mediterranean, 1 (2004)

3. W. Li, F. Gu, A. D. Ball, C. E. Phipps, Mechanical Systems and Signal Processing, 15 (2001)
4. A. Ypma, A. Leshem, R. P.W. Duin, Neurocomputing, 49 (2002)

5. G. Gelle, M. Colas, G. Delaunay, Mechanical Systems and Signal Processing, 14 (2000)

6. J. Antoni, Mechanical Systems and Signal Processing, 19 (2005)

7. W. Lu, J.C. Rajapakse, IEEE Trans. Neural Networks, 16 (2005)

8. W. Lu, J.C. Rajapakse, Neurocomputing 69 (2006)

9. S. Choi, A. Cichocki, Electronics Letters, 37 (2001)

10. C.J. James, O.J. Gibson, IEEE Trans. Biomed. Eng. 50 (2003)

11. R.B. Randall, J. Antoni, S. Chobsaard, Mechanical Systems and Signal Processing, 15 (2001) 